\title{
Study on the muon lateral distribution based on the first stage of LHAASO-KM2A
}

\author{
Guang-Guang Xin ${ }^{1, a, b}$, Yi Zhang $^{\mathrm{b}}$, You-Liang Feng ${ }^{\mathrm{c}}$,Shiping Zhao ${ }^{\mathrm{b}}$,Ying-Ying Guo ${ }^{\mathrm{b}}$, \\ Gang $\mathrm{XiaO}^{\mathrm{b}}$, Cong $\mathrm{Li}^{\mathrm{b}}$,Xiong Zuo ${ }^{\mathrm{b}}$, Ling-Yu Wang ${ }^{\mathrm{b}}$,Ning Cheng ${ }^{\mathrm{b}}$, on behalf of the \\ LHAASO Collaboration

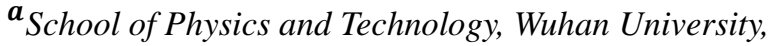 \\ Wuhan, 430072, China \\ ${ }^{\boldsymbol{b}}$ Key Laboratory of Particle Astrophysics, Institute of High Energy Physics, Chinese Academy of \\ Sciences, \\ Beijing, 100049, China \\ ${ }^{c}$ Key Laboratory of Dark Matter and Space Astronomy, Purple Mountain Observatory, \\ Chinese Academy of Sciences, \\ Nanjing ,210034, China \\ E-mail: xingglihep.ac.cn; zhangyieihep.ac.cn; fengylepmo.ac.cn
}

\begin{abstract}
The first stage of LHAASO-KM2A has been completed in Sichuan, China. KM2A is composed of electromagnetic particle detectors (ED) and muon detectors (MD), focusing on gamma ray astronomy above $30 \mathrm{TeV}$ and cosmic ray physics in the "knee" region. The ED array in the first stage is installed into two clusters and the distance between the two clusters is around 230 meters. The MDs are distributed in one of the clusters. Each MD is covered with soil of 2.5 meters thick. We analyzed the lateral distribution of muons which are 140-300 meters away from Shower axis, and the primary cosmic ray energy is about $100 \mathrm{TeV}$.
\end{abstract}

36th International Cosmic Ray Conference -ICRC2019-

July 24th - August 1st, 2019

Madison, WI, U.S.A.

\footnotetext{
${ }^{1}$ Speaker

(C) Copyright owned by the author(s) under the terms of the Creative Commons 


\section{Introduction}

The secondary muons in the extensive air showers (EAS) carry abundant essential information of primary cosmic rays (CR) particles. Most of muons, which are most abundant products of hadronic interactions in the EAS, come from decay of pions, kaons, and particles containing heavy quarks. The number of muons is therefore used to estimate the CR mass by relating to the measured electromagnetic energy. And it is also an efficient parameter to distinguish gamma rays from overwhelming CRs since there are far fewer muons in the gammaray showers.

Measurement of the lateral distribution of muon density can provide additional information for studying the mass composition of the primary CR and test the hadron interaction model. The muon density in a hadronic shower decreases gradually with the lateral distance to the shower core, but the distribution is flatter than that of electromagnetic particles, which can be described by the NKG function. Uncertainties is inevitable in calculating the hadronic shower development and the muon lateral distribution, due to the limitation of perturbative quantum chromodynamics.

There have been several attempts to measure the lateral distribution of muons with energies greater than $100 \mathrm{TeV}$. The MACRO and IceCube experiments measured the lateral distribution of muons from the center of the shower to estimate the transverse momentum of the muon parent. The KASCADE-Grande experiment has measured lateral distributions of muons which are 100-610 meters away from shower axis at energy from $10^{16} \mathrm{eV}-10^{17} \mathrm{eV}[1]$. The YBJ-HA experiment reported a measurement of lateral distribution of muons with the lateral distance ranging from $20 \mathrm{~m}$ to $100 \mathrm{~m}$ above $100 \mathrm{TeV}$ and they carefully accounted for the punch-through effect via simulation. [2]

The Large High Altitude Air Shower Observatory (LHAASO) is a hybrid array for very high-energy gamma ray astronomy and cosmic ray physics. The one $\mathrm{km}^{2}$ array (KM2A) is one of the main parts of LHAASO, covering an area of $1.3 \mathrm{~km}^{2}$ to detect gamma rays at energy from $10 \mathrm{TeV}$ to $1 \mathrm{PeV}$. A quarter of the whole KM2A has been constructed in the summer of 2019. The first stage of KM2A, with 71 electromagnetic detectors ( ED ) and 10 muon detectors ( MD ) has been in stable operation for half a year.

In this work, we utilize the data obtained by the first stage of KM2A to study the lateral distribution of muons with primary energy above 100TeV. EDs are installed into two clusters which are shown in Fig.1 and the distance between these two clusters is 230 meters while MDs are distributed in the southern cluster. EDs are used to reconstruct the core, direction and energy of showers. We take advantage of the large distance between the two clusters to analyze the 
lateral distribution of muons which are around 200 meters away from shower axis. And the "punch through" effect from high-energy electromagnetic particles can be neglected in this case.

\section{Experiment}

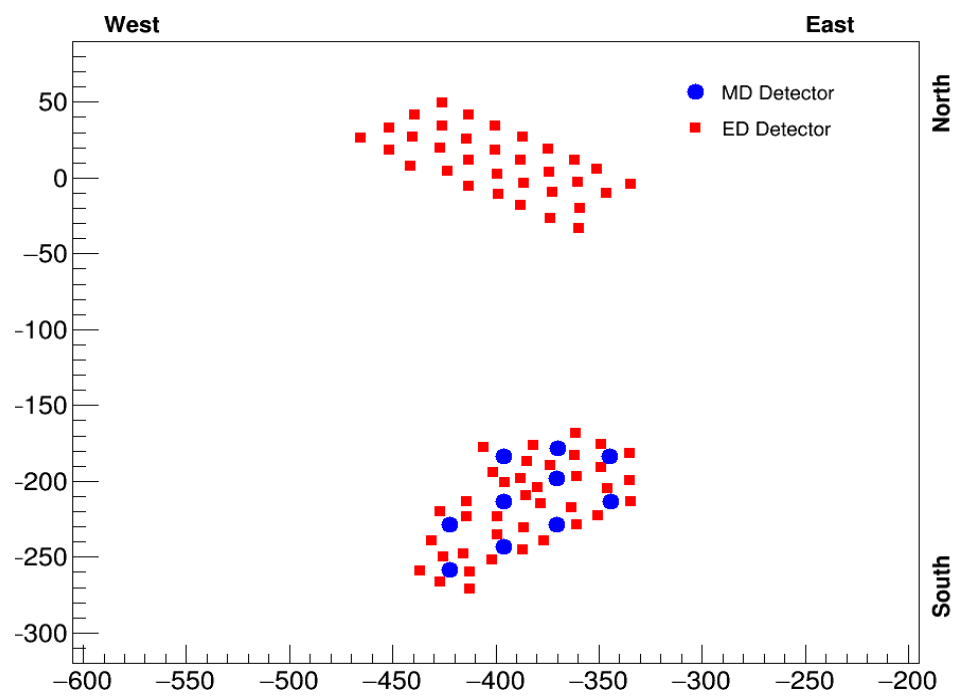

FIGURE. 1. Layout of arrangement for first stage of LHAASO-KM2A. Blue circles and red rectangles correspond to MDs and EDs respectively.

The first-stage array, which is about $2 \%$ of the size of $\mathrm{KM} 2 \mathrm{~A}$, was constructed at the LHAASO site, at the altitude of $\sim 4400 \mathrm{~m}$. It had been in stable operation since December, 2018. This detector array consists of 71 EDs and 10 MDs. The ED array is divided into two clusters, with 38 in the south cluster and 33 in the north cluster. In each cluster, EDs are uniformly distributed with the same spacing of $\sim 15 \mathrm{~m}$. The ten MDs are placed in the south cluster. The layout of the arrangement for this array is shown in Fig.1.

The ED is a scintillator detector with area of $1 \mathrm{~m}^{2}$, utilizing wave-length shifting fibers to deliver scintillation light to Photomultiplier ( PMT ) [3]. The time resolution of the ED module is less than $2 \mathrm{~ns}$. The ED is able to detect single particles with a resolution of $25 \%$, and measure 10000 particles with a resolution better than $5 \%$.

The MD is a water Cherenkov detector with an effective area about $36 \mathrm{~m}^{2}$. The tank is under shielding of $2.5 \mathrm{~m}$ thick overburden soil to prevent low energy electromagnetic particles from entering the water. Thus, the threshold for muon is about one GeV. The dynamic range for each MD can be up to 10000 particles. The time resolution of the MD is about $10 \mathrm{~ns}$, and the charge resolution for a single muon is better than $25 \%$. Details about the MD are described in [4].

The trigger signal is provided by the ED array when more than 5 EDs are fired simultaneously in a time window of $200 \mathrm{~ns}$. When the trigger signal is generated, all signals over the threshold from both EDs and MDs in a time window of $10 \mathrm{~ns}$ are acquired and stored for off-line analysis. 


\section{Simulation}

The development of the air shower in the atmosphere and the interaction of secondary particles into detectors are well simulated by the KM2A standard simulation chain [5]. The configuration of the ED unit detector basically takes the size and structure of the actual ED detector, including aluminum housing, lead plate, Tevk reflective paper, surface-slotted PMT, scintillator and wavelength shifting fiber. The MD unit detector basically takes the size and structure of the actual MD detector. The top of the detector is a trapezoidal cylindrical soil layer with $2.5 \mathrm{~m}$ in thickness and the bottom is a cylindrical soil layer with a diameter of $13.9 \mathrm{~m}$. Concrete tanks, water liners, water and PMTs are placed in the soil layer.

The development of EAS in the atmosphere is generated by the CORSIKA package [6] with version number 76400. We use QGSJET2 [7] and GHEISHA [7] for the high and low energy hadronic interaction models, respectively. Different mass of primaries $(\mathrm{H}, \mathrm{He}, \mathrm{CNO}, \mathrm{MgAlSi}$ and $\mathrm{Fe}$ ) are generated in the energy range from $0.1 \mathrm{TeV}$ to $10 \mathrm{PeV}$ according to a power law spectrum with an index of -2.7 . Zenith angle is $0-70^{\circ}$, and azimuthal angle is $0-360^{\circ}$. Observation level is $4410 \mathrm{~m}$. All events are generated in a uniform shower core distribution, scattering in a circle around the array center with the radius of $600 \mathrm{~m}$, to ensure a large enough sampling area containing almost all events that can trigger the array. These events are properly weighted to resemble the spectra of two CR component models: the EPOS model and the Fluka model .

The performance of this simulation package is discussed in [5].

\section{Analysis}

This analysis is based on the data collected in the period from December 2018 to May 2019. Events are selected by imposing the following five criteria on the reconstructed data:

1) Each shower event should fire four or more detectors.

2) The sum of the number of particles from all fired detectors should be larger than 100 and less than 300 .

3) The reconstructed zenith angle of the shower should be less than 15 degrees.

4) The position of the reconstructed shower core should be in the north array.

5) The number of fired detectors in the north array should be no less than 10 , and the number of fired on the south side of the detectors is less than $1 / 8$ of that in the north side.

The experiment data are consistent with the MC data when imposing above 5 criteria. Comparison of essential parameters are listed. The total number of particles detected by the ED array $\left(\sum \rho\right)$ is related to the energy of this primary CR. This parameter can be a good indicator for the true energy of the CRs. [8] Fig.2 shows the distribution of $\sum \rho$ from the experiment and 
from MC simulation. The black points are the experiment data while the red points indicate the MC sets.

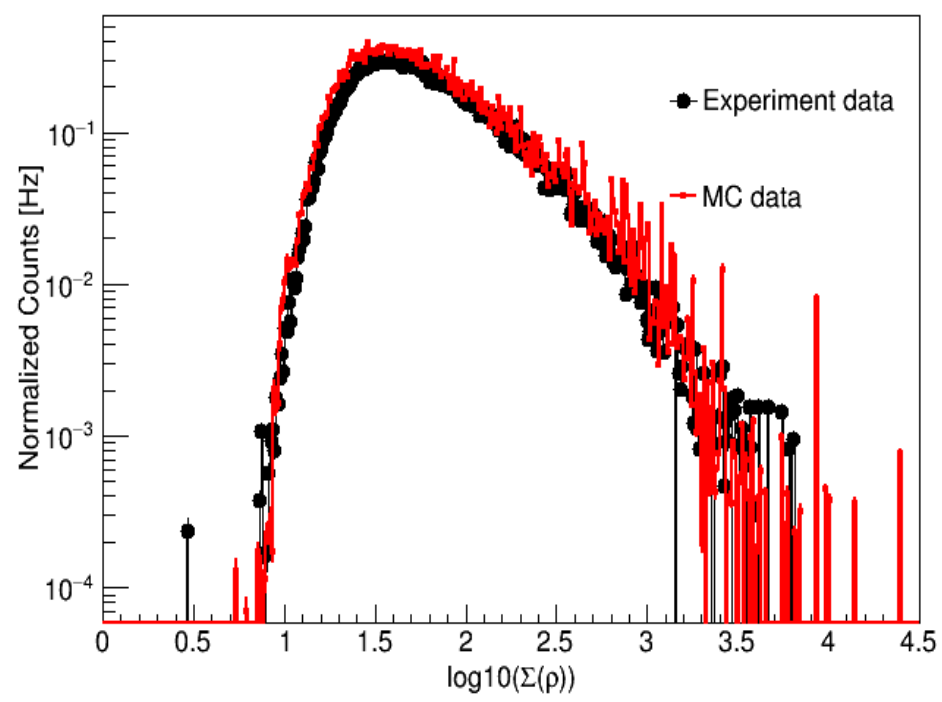

FIGURE. 2. The distribution of the sum of particle density for all detectors $\left(\sum \rho\right)$ in the experiment and $\mathrm{MC}$ simulation.

Measurements of the lateral distribution of muon would be sensitive to the resolution of the shower core position. With respect to the shower core, lateral distribution of secondary particles in an electromagnetic shower can be described by the NKG function. At the same time, this function then can be used to reconstruct the shower core by fitting the particle density at different distances. The left panel of Fig.3 shows the distribution of the core difference between the real core and reconstructed core. The position resolution is defined that the confidence region contains $50 \%$ of the events. By this method, the position resolution in our experiment is 5.5 meters. The right panel of Fig. 3 shows that the primary energy is mainly distributed around $100 \mathrm{TeV}$, after applying the above criteria.
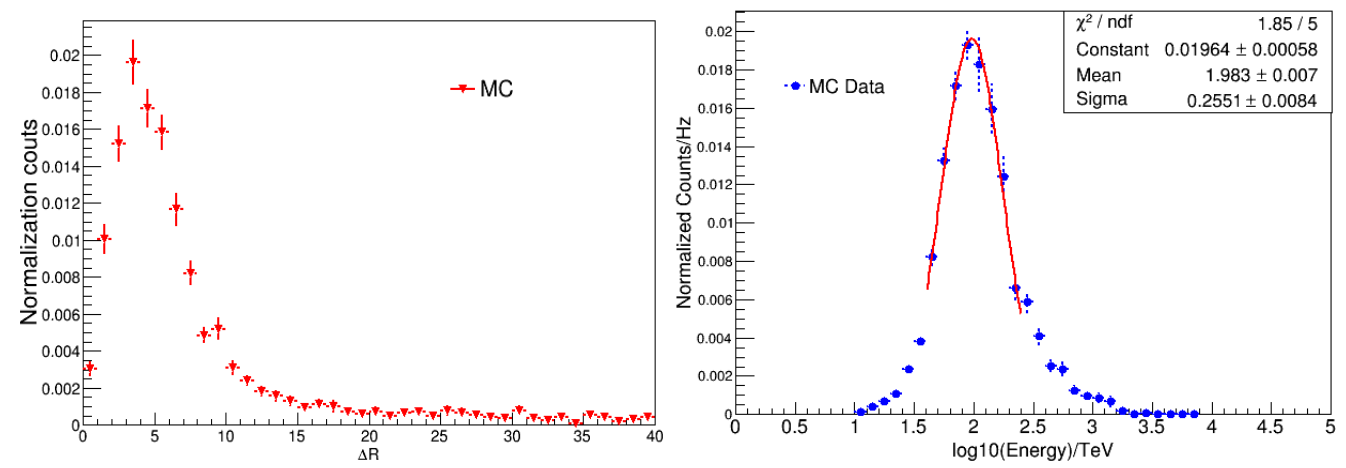

FIGURE. 3: Left: The difference between the real core position and the reconstructed core position. Right: After imposing above 5 criteria,the primary energy is mainly distributed around $100 \mathrm{TeV}$. 
Discrimination of the shower muons and the background muons from other irrelevant showers is important in this work. A cut on the time residual of muons is applied. The time residual distribution of the muons is present in Fig.4 left panel. The black points are the experiment data and the red points are from MC. The peak indicates the muon signal in the air shower, and the width represents the time resolution of the detector. The platforms on both sides come from the background muons. Therefore, the muons with time residual in the range from $-20 \mathrm{~ns}$ to $40 \mathrm{~ns}$ are selected in our analysis. According to the time window and the background platform, survived background events are only $0.002 \mathrm{~Hz}$ for each detector.
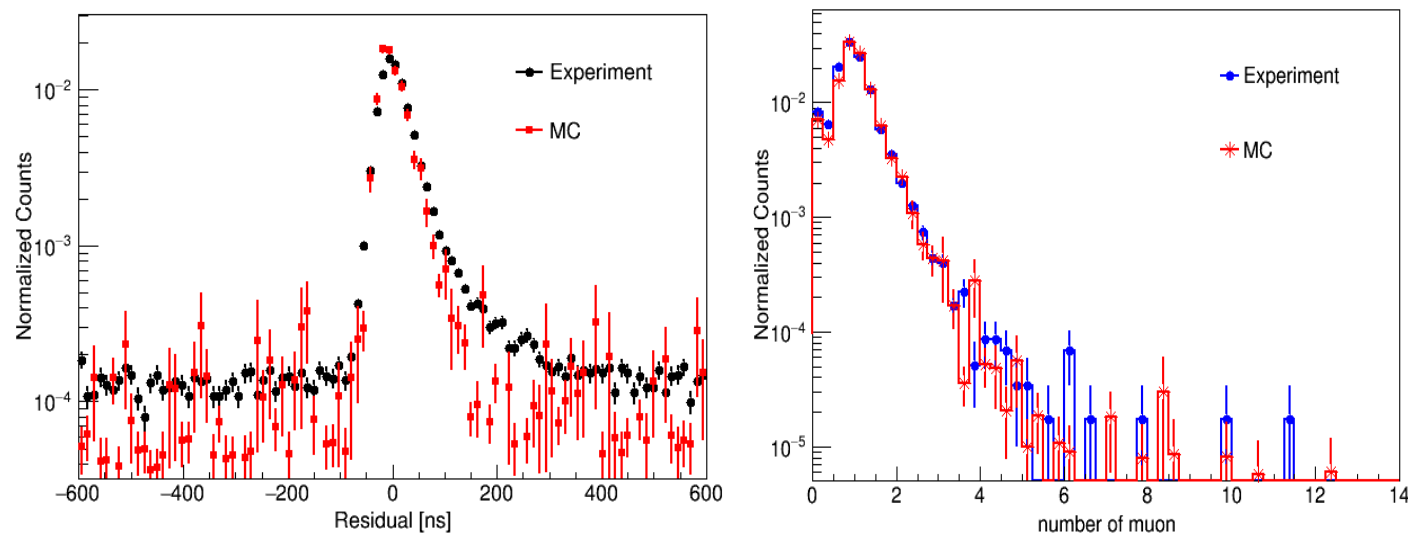

FIGURE. 4: Left: The distribution of the time residual of the muon detector. Right: The muon spectrum of MD.

After applying above criteria, the spectrum of the selected muons is shown in Fig.4. The blue points are the experimental data and the red points are from MC simulation. Apparently, the good agreement between the experiment and the simulation indicates that our data is well understood.

\section{Results}

Events are grouped by the distance between the position of a MD unit and the shower axis in a step of 10 meters. For the group of $R_{i}$, the average number of muons $\rho\left(R_{i}\right)$ in a shower is then calculated as:

$$
\rho\left(R_{i}\right)=\frac{M_{i}}{K_{i}} \times \frac{1}{S \cdot \cos \theta} \quad,
$$

where $M_{i}$ is the number of muons in the i-th group;

$\mathrm{K}_{\mathrm{i}}$ is the number of events in the $\mathrm{i}$-th group;

$\mathrm{S}$ is the effective area of the detection unit of the MD, which is $36.3 \mathrm{~m}^{2}$ in our case;

$\theta$ is the zenith angle of the CR event. 
We mainly focus on the lateral distribution of muons in case that the core positions are in the north array. Because the MD detector is far from the core, we do not consider the effect of the "punch through" effect in this work. Due to the different positions of the $\mathrm{MD}$, the experiment can observe the distribution of muons at different distances from the core. Different detectors get similar results at the same distance from the core, so we can combine the results that measured by different detectors at the same distance.

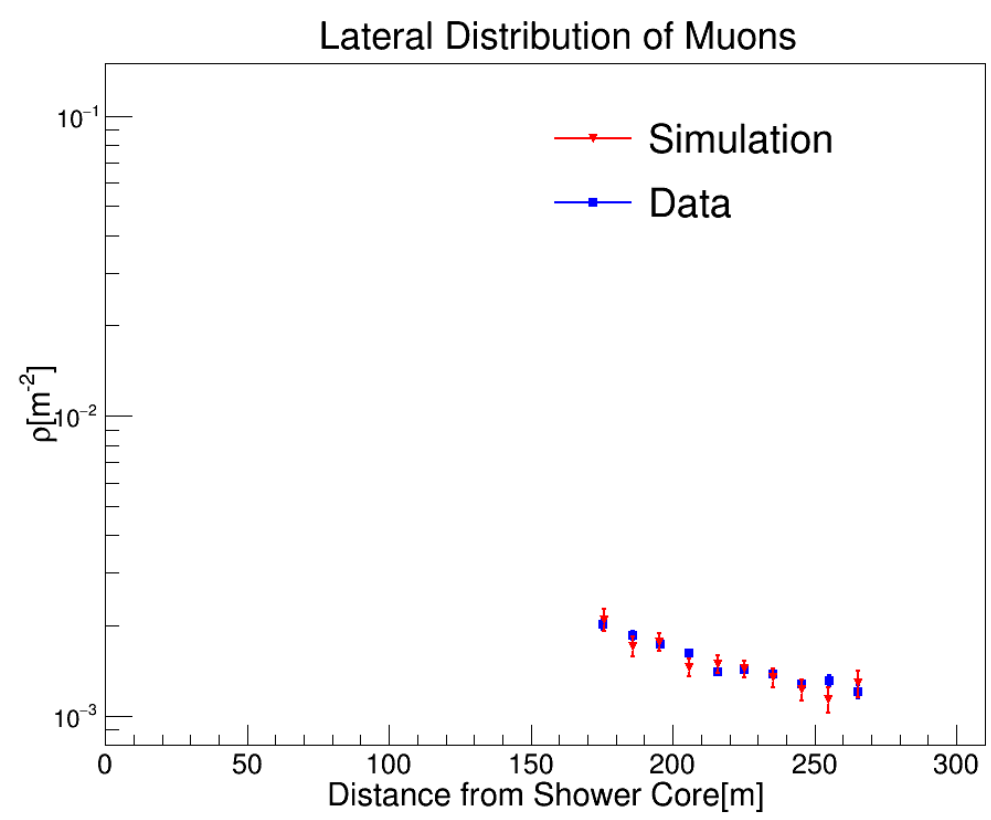

FIGURE. 5. Measured muon density distribution with zenith angle of the shower $\theta<15^{\circ}$. The blue square represent muon density distribution obtained from experimental data and the red points represent the result of the MC simulation.

Fig.5 shows the measured lateral distribution of muons around $100 \mathrm{TeV}$ with zenith angle less than 15 degree. The blue squares represent muon density distribution obtained from experiment data, while the red points represent the distribution from MC simulation. This experiment result is a combination of the results of eight well-running MDs. The overall trend of the lateral distribution of muons is decreasing. Apparently, the experiment is in good agreement with simulation. Accurate measurement of muon distribution can effectively distinguish primary hadrons and primary gamma, which is of great help to the study of gamma astronomy.

\section{Summary}

The simulated data are consistent with the experiment. The lateral distribution of muons is measured in this work for primary CR energy around $100 \mathrm{TeV}$ at distance around $200 \mathrm{~m}$. 
Because a quarter of the MD array has been successfully deployed, a detailed analysis on the lateral distribution of muons would be implemented soon.

\section{Acknowlegements}

This work is partly supported by the National Key R\&D Program of China No. 2018 YFA0404202 and National Natural Science Foundation of China under Grant 11775233.

\section{References}

[1] W.D. Apel, J.C. Arteaga-Velázquez, K. Bekk, et al. Lateral distributions of eas muons measured with the kascade-grande muon tracking detector in the primary energy range 1016-1017ev. Astroparticle Physics, 65:55-63, 2015.

[2] You-Liang Feng, Yi Zhang, et al, Lateral distribution of EAS muons measured at the primary cosmic ray energy around 100TeV, Chinese Physics C Vol. 37, No. 1 (2019) .

[3] ZHAO Jing ,LIU jia ,SHENG Xiang-Dong, et al, Design and performance of electromagnetic particle detector for LHAASO-KM2A, Chinese Physics C Vol. 38, No. 3 (2014).

[4] Zuo Xiong, Xiao Gang, Feng Shao Hui, et al. Design and performances of prototype muon detectors of LHAASO - KM2A, Nuclear Instruments and Methods in Physics Research A,2015.

[5] Songzhan Chen, et al, Detector simulation of LHAASO-KM2A with Geant4, PoS(ICRC2017) .

[6] D. Heck, J. Knapp, J. N. Capdevielle, G. Schatz, and T. Thouw. CORSIKA: a Monte Carlo code to simulate extensive air showers. February 1998.

[7] S. Ostapchenko. Qgsjet-ii: towards reliable description of very high energy hadronic interactions. Nuclear Physics B - Proceedings Supplements, 151(1):143-146, 2006.

[8] M. Amenomori and Tibet As $\gamma$ Collaboration. Performance of the Tibet air shower array for search for gamma-ray point sources. International Cosmic Ray Conference, 2:704-707, August 1991. 